\title{
An Efficient Method of Power Spectral Density Estimation for On-Chip IR-UWB Transmitter Self-Calibration
}

\author{
Anthony Goavec, Mykhailo Zarudniev, Rémy Vauché, Member, IEEE, Frédéric Hameau, \\ Jean Gaubert, and Eric Mercier
}

\begin{abstract}
In ultra-wideband impulse radio communications, process, voltage, and temperature variations may impact the operation of emitters by modifying the emitted power spectral density. In order to compensate the impact of such variations and to respect the spectral emission masks, self-calibration systems able to estimate the output power spectral density have to be integrated in ultra-wideband impulse radio transmitters. In this paper, a new power spectral density estimation method for ultra-wideband impulse radio signals is presented. Unlike the discrete Fourier transform classically used to estimate the power spectral density, the proposed method, which is based on the measurement of the time varying envelope and instantaneous frequency of the emitted pulse, preserves a link between the time and the frequency domain thanks to a piece-wise approximation of these waveforms. This time-to-frequency link enables a straightforward pulse shape control by using simple control methods and allows to comply with spectral masks required by ultra-wideband communication regulations and standards such as the IEEE 802.15.4a. Thus, both envelope and instantaneous frequency extraction techniques are introduced for acquisition of these time domain waveforms in ultra-wideband impulse radio domain. The proposed techniques are validated and allow to consider future hardware implementation in order to build onchip calibration systems for ultra-wideband transmitters.
\end{abstract}

Index Terms-Fourier transform, impulse radio, instantaneous frequency, power spectral density, transmitter calibration, ultrawideband.

\section{INTRODUCTION}

$\mathbf{F}$ OR ABOUT A decade, ultra-wideband (UWB) technology has demonstrated its potential for high [1] and low [2] data rate short range wireless communications [3], [4]. Moreover, it offers high localization performances by accurate

Manuscript received April 25, 2016; revised July 19, 2016 and September 6, 2016; accepted September 30, 2016. Date of publication November 8, 2016; date of current version February 23, 2017. This paper was recommended by Associate Editor C. Li.

A. Goavec is with Univ. Grenoble Alpes, F-38000 Grenoble, France and also with CEA, LETI, MINATEC Campus, F-38054 Grenoble, France and also with the Aix Marseille Université, CNRS CNRS, Université de Toulon, IM2NP UMR 7334 Marseille, France (e-mail: anthony.goavec@cea.fr).

M. Zarudniev, F. Hameau, and E. Mercier are with Univ. Grenoble Alpes, F-38000 Grenoble, France and also with CEA, LETI, MINATEC Campus, F-38054 Grenoble, France (e-mail: mykhailo.zarudniev@cea.fr; frederic.hameau@cea.fr; eric.mercier@cea.fr).

R. Vauché and J. Gaubert are with the Aix Marseille Université, CNRS, Université de Toulon, IM2NP UMR 7334 Marseille, France (e-mail: remy.vauche@univ-amu.fr; jean.gaubert@univ-amu.fr).

Color versions of one or more of the figures in this paper are available online at http://ieeexplore.iee.org.

Digital Object Identifier 10.1109/TCSI.2016.2617887

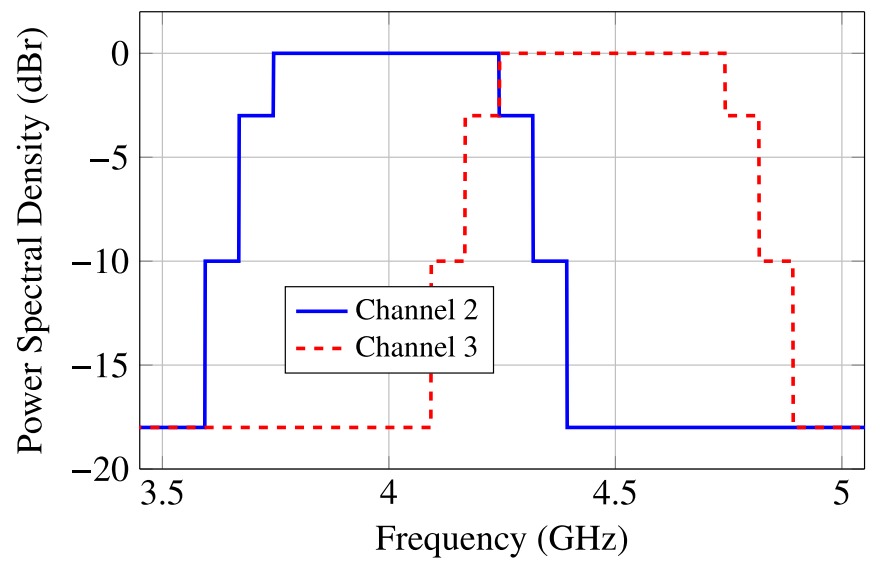

Fig. 1. Frequency constraints for two adjacent channels described in 802.15.4a IEEE Standard.

measurement of the time of flight thanks to its robustness to multipath fading [5]. In 2002 the Federal Communications Commission (FCC) defined an UWB signal with a minimum $-10 \mathrm{~dB}$ bandwidth of $500 \mathrm{MHz}$ or $-10 \mathrm{~dB}$ fractional bandwidth of $20 \%$ [6]. To enable the coexistence and dissemination of UWB systems, the FCC and the European Telecommunications Standards Institute (ETSI) set power emission constraints for all kinds of UWB devices operating in the 3.1 to $10.6 \mathrm{GHz}$ UWB frequency band. Inside the band, the mean power spectral density is limited to $-41.3 \mathrm{dBm} / \mathrm{MHz}(0 \mathrm{dBm} / 50 \mathrm{MHz}$ for the peak power spectrum density).

To establish communications between UWB devices, the IEEE 802.15.4a Standard [7] defines 15 channels with a $-3 \mathrm{~dB}$ bandwidth of $499.2 \mathrm{MHz}, 1081.6 \mathrm{MHz}$, and $1331.2 \mathrm{MHz}$. A transmission power spectral density (PSD) mask is connected to each IEEE channel as shown in Fig. 1 for two adjacent channels among the 15 possible. Considering the high required bandwidths, two adjacent IEEE channels have very close central frequencies. For instance, channels 2 and 3 of this standard are centered on $3993.6 \mathrm{MHz}$ and $4492.8 \mathrm{MHz}$ respectively, which in combination with the emission masks imply challenging power limits for each channel. For the two channels shown in Fig. 1, to insure that the PSD complies with IEEE 802.15.4a Standard, the required rejection is $10 \mathrm{~dB}$ and $18 \mathrm{~dB}$ at $\pm 324 \mathrm{MHz}$ and $\pm 398 \mathrm{MHz}$ offsets relative to the central frequency, respectively. Note that the power limit 
in Fig. 1 is given in $\mathrm{dB}$ relative to the maximum of the power spectral density of the emitted signal.

In order to generate a signal with ultra-large bandwidth, a way is to use impulse modulation of short pulses instead of continuous wave carrier modulation, since this allows to reduce the complexity and the cost of the transmitter. However, impulse radio (IR) circuits are disturbed by process-voltagetemperature (PVT) variations that cause performance degradations, impacting notably the emitted pulse PSD shape and amplitude [8]. Therefore, to comply with the spectral masks of the IEEE 802.15.4a Standard shown in Fig. 1 in the presence of PVT variations is not a trivial task.

The computation of the PSD of the signals emitted by IR systems has been largely studied [9]-[11]. For instance, for a linear modulation of an impulse signal considered as a random process, it is possible to use the Bennett's formula [12] which shows that the PSD is directly proportional to the square modulus of the Fourier transform of the emitted pulses. However, analytical determination of the Fourier transform of the pulse used to create the IR-UWB signal is difficult. The conventional solution consists of performing the discrete Fourier transform (DFT), but it requires an analog-to-digital conversion of the radio frequency (RF) signal with a very high cost from the hardware implementation point of view. Moreover, in order to be able to change the PSD values in order to respect the IEEE standard masks, a practical manner is to work in the time domain, but it is difficult to understand the impact of time domain modifications in the DFT formula. Thus the time-to-frequency link is a common topic of study in the literature with the aim of computing the Fourier transform.

Firstly some authors have focused their attention on pulse shapes that have an analytical representation in order to find good candidates respecting spectral masks by Fourier transform computation. In this context, the Gaussian pulse and its high-order derivative have largely been studied [13]-[15] since they are comfortable in terms of Fourier transform computation. Other signals have been studied such as Hermite polynomials or prolate spheroidal functions [16], [17]. Pulse design methods also exist based on the linear combination of the above-mentioned signals [18]-[20]. The superposition of Gaussian sub-carriers used in [21] and [22] enables flexibility for the realization of time domain pulse shapes in order to generate spectra which fits on FCC masks. The use of analytical pulse shapes allows to have a good knowledge on the spectrum but places a lot of constraints in the design of electronic devices required for the generation of such waveforms.

Some circuits have been realized in order to generate the pulse shapes previously mentioned, as in [23] where a fifth derivative Gaussian pulse generator is presented. But the aim in the design of such transmitters is often to comply with mean power limits defined by the FCC. The effect of PVT variations on the PSD is generally not a problem to respect these limits because the boundaries are far from the central frequency. Moreover, with the aim of generating the pulse shapes previously mentioned based on mathematical functions, the hardware implementation of emitters may be impossible from designers' point of view. The aim of this study is to

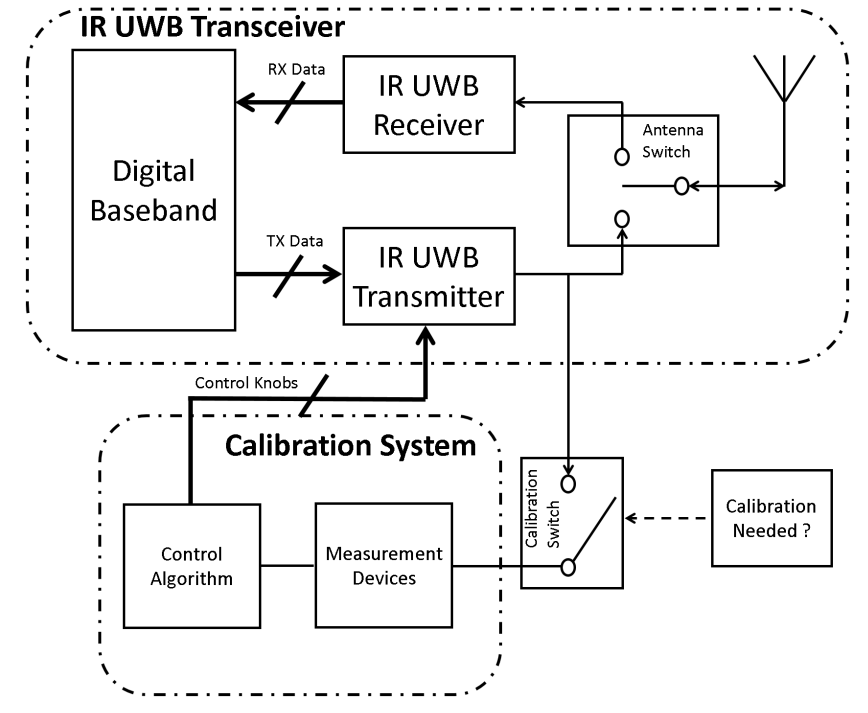

Fig. 2. Positioning of a PSD Calibration System in an IR-UWB Transceiver.

obtain an IR-UWB signal compliant with the IEEE 802.15.4a Standard masks.

The trivial solution consists on adding filters that limit the spectral emissions at particular frequency points independently of the impact that the PVT variations may have on the emitted pulse PSD shape. However, instead of using such costly hardware filters, it could be interesting to build smart on-chip calibration system for IR-UWB transmitters which have often a lot of control possibilities to guarantee that the pulse PSD fits on the emission mask [24]. In Fig. 2 such an IR-UWB transceiver is represented. A single antenna is shared by the transmitter (TX) and the receiver (RX). A digital baseband unit is often used in order to process the data. On the transmitter side, a calibration loop is used in order to adjust the transmitter emission before the antenna when the TX is in calibration mode. This loop could be disconnected from the transceiver thanks to a calibration switch in order to not disturb its operation in the normal TX mode. Then information would be extracted from the output of the transmitter and a control method should act on the possible control knobs of the transmitter in order to calibrate the PSD.

To build such an integrated calibration system, it is proposed in this work a generic way to approximate the impulse radio signal in time domain by a simpler representation in order to estimate the frequency domain characteristics of the signal on a digital embedded unit, and make it compliant with the settings of the IEEE 802.15.4a Standard. Also this work is limited to the study of impulse radio signal with a maximum $-3 \mathrm{~dB}$ bandwidth of $1331.2 \mathrm{MHz}$, which implies a relative pulse duration of a few nanoseconds. In [25], a "modifiedtriangular" shape was proposed as an alternative to build an IR transmitter with controllable PSD emission. It is an example of a very simple shape which allows analytical computation of the Fourier transform that makes it possible to modify the pulse parameters in a simple way in order to have a time domain signal respecting the IEEE 802.15.4a Standard frequency mask. Conversely, the method proposed in this work can be 


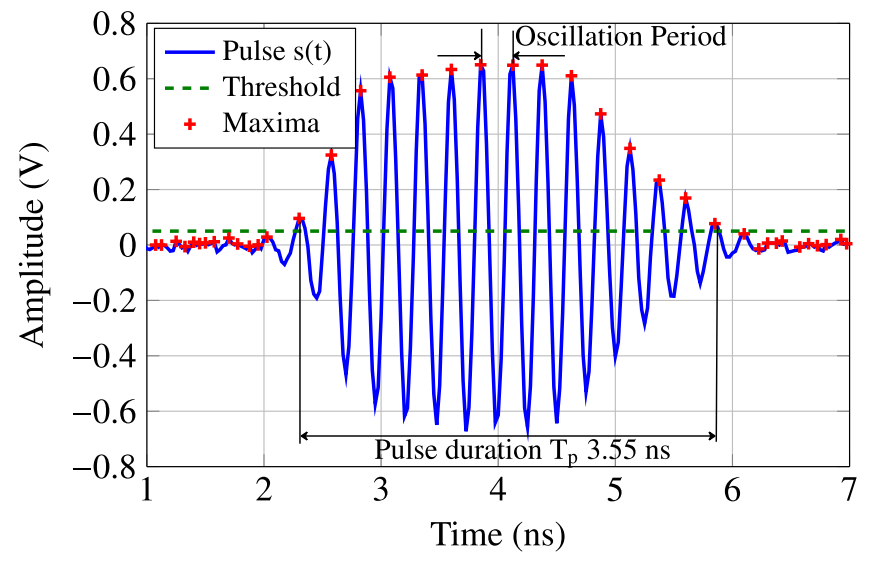

Fig. 3. Time domain measurement of the UWB pulse.

applied to all kinds of impulse radio signals because it is based on the measurements of the envelope and the instantaneous frequency of the radiofrequency signal. This study wants to prove that it is possible to use these well-known continuous wave techniques in order to extract baseband information of IR-UWB signals. Moreover it is conceivable to measure these waveforms on-chip, which enables the development of embedded calibration systems.

This paper is organized as follows. In Section II, the time domain signal model based on the envelope and the instantaneous frequency waveforms is presented. In order to extract these time domain waveforms from the emitted signal, a down-conversion method and an RF signal based method are introduced in Section III. A piece-wise approximation is employed on the envelope and the instantaneous frequency in Section IV in order to finally establish a frequency representation of the Fourier transform of the pulse. Section V shows the validity of the presented method by applying it to an IR-UWB signal and demonstrating its use in transmitter calibration.

\section{Signal Modeling}

In this section, a convenient model for the UWB signal is introduced. As seen before, a common hypothesis for IR-UWB emitter is to use the real valued Gaussian pulse model in order to describe its time domain behavior and to enable Fourier transform computation [13]-[15]. In practice, it does not seem to be realistic to find some mathematical functions representing adequately the output signal of existing emitters in the frequency domain by using a reduced set of parameters.

Indeed, the signal can be characterized easily in the time domain. In Fig. 3 an example of a measured impulse signal is shown. It is used in an existing IR-UWB transmitter device [26] for localization applications. From the figure, it becomes clear that it is difficult to approximate the envelope of the RF signal by any existing simple mathematical function, at least since the rise and fall of the pulse can have a lot of different shapes.

The second observation concerns the behavior of the downconverted RF signal. The PSD for an impulse signal is often noticed asymmetrical compared to its carrier frequency axis (as shown in Fig. 4, where $S(v)$ is the spectral part for positive

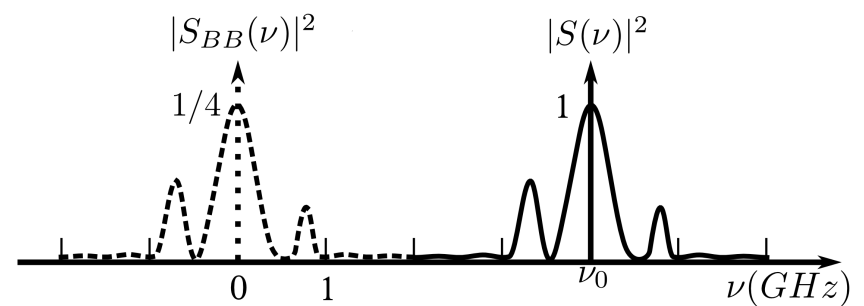

Fig. 4. The UWB signal in frequency domain.

frequencies of RF signal $s(t)$ ). The PSD $S_{B B}(v)$ is this same spectral part down-converted in baseband with a frequency translation $\delta_{\nu_{0}}$. However, the PSD can not be asymmetrical if the time domain signal is actually a function with real values.

So, because of the contraposition of the Fourier transform property, the signal $s_{B B}(t)$ can be seen as a complex valued function. This statement increases the difficulty to find an existing function approaching the impulse signal.

Following these two observations, the baseband signal $s_{B B}(t)$ can be written as follows:

$$
s_{B B}(t)=a(t) \mathrm{e}^{j \varphi(t)},
$$

where $a(t)$ and $\varphi(t)$ are respectively the envelope and the instantaneous phase across the representation of band-pass signal [12]. It is assumed that the signal $s_{B B}(t)$ complies with the definition of band-pass signal. For that, it is considered (1) makes sense only if its bandwidth is negligible compared to its carrier frequency $v_{0}$. In other words, the studied signals are limited to UWB signals whose bandwidth does not produce power interferences between the $\nu_{0}$ centered signal and its baseband image as shown in Fig. 4. Moreover, signals with, for example, dual carriers cannot be studied here [27].

Note that in this work, the carrier frequency $v_{0}$ is considered as the inverse of the mean value of all oscillation periods in the pulse (see Fig. 3). To estimate the pulse duration only the oscillations with a local maximum that are higher than a threshold level are taken into account. Here the threshold has been chosen with a value of $50 \mathrm{mV}$ in order to differentiate the first effective oscillation in the pulse from the non-true oscillations due to electronical noise possibly existing where the pulse is turned off. If a noisy oscillation was taken into account, the effective duration of the pulse would be wrong and the estimated carrier frequency too.

To meet with the model adopted in (1), the signal $s(t)$ is written as:

$$
s(t)=\Re\left(s_{B B}(t) \mathrm{e}^{j 2 \pi v_{0} t}\right) .
$$

From this equation, it is possible to write,

$$
s(t)=a(t) \cos \left(2 \pi v_{0} t+\varphi(t)\right) .
$$

From (2) and (3), the function $s(t)$ is actually a finite real envelope $a(t)$ up-converted to the central frequency $v_{0}$ and modulated by the instantaneous phase $\varphi(t)$. This analysis can also be found in (1) where $\varphi(t)$ gives its complex nature to the baseband signal. By assuming that the instantaneous phase is a differentiable function, it is possible to express the 
instantaneous frequency as follows:

$$
f(t)=\frac{1}{2 \pi} \frac{d \varphi}{d t}(t)
$$

In further sections, these time parameters $a(t)$ and $f(t)$ are used in order to establish a frequency representation of the signal and also to approach the analytical Fourier transform result. For this purpose, three main points will be discussed:

- Extraction of the time domain waveforms $a(t)$ and $f(t)$ in (3) and (4);

- Establishment of analytical frequency representation of the signal using the time domain waveforms;

- Perspective of the PSD modification using $a(t)$ and $f(t)$ controls in the time domain thanks to the established time-to-frequency link.

\section{Extraction of the Time Domain Parameters}

This section introduces the extraction of the time domain waveforms. Here, two methods are presented in order to obtain the envelope $a(t)$ and the instantaneous frequency $f(t)$ from RF signal $s(t)$.

\section{A. Down-Conversion Method}

This method is based on the down-conversion of the emitted signal in order to mathematically compute $a(t)$ and $f(t)$ in the frequency baseband.

Equation (3) can be rewritten as:

$$
s(t)=a(t)\left(\frac{\mathrm{e}^{j\left(2 \pi \nu_{0} t+\varphi(t)\right)}+\mathrm{e}^{-j\left(2 \pi v_{0} t+\varphi(t)\right)}}{2}\right) .
$$

So the ideal down-conversion of $s(t)$ using the multiplication by $\mathrm{e}^{-j\left(2 \pi v_{0} t\right)}$ in time domain gives:

$$
s(t) \mathrm{e}^{-j\left(2 \pi v_{0} t\right)}=\frac{s_{B B}(t)+s_{H R F}(t)}{2},
$$

where

$$
s_{B B}(t)=a(t) \mathrm{e}^{j \varphi(t)}
$$

and

$$
s_{H R F}(t)=a(t) \mathrm{e}^{j\left(2 \pi 2 \nu_{0} t+\varphi(t)\right)} .
$$

By filtering $s_{H R F}(t)$ all the needed information are contained in the real and the imaginary parts of the estimated baseband signal $s_{B B}(t)$, respectively obtained on I and Q paths (Fig. 5).

The ideal estimation of the envelope and the phase in time domain parameters also consists of the computation of the modulus and the argument of the baseband signal. The derivative of $\varphi(t)$ gives the instantaneous baseband frequency $f(t)$ as seen in (4).

\section{B. RF Signal Based Method}

RF signal based method allows the estimation of the envelope and the instantaneous frequency with the help of on-chip analog operations directly applied on the RF signal.

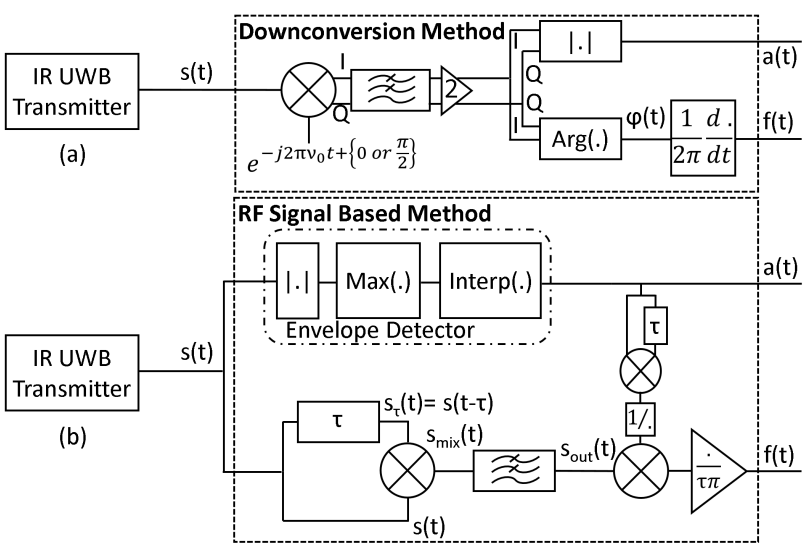

Fig. 5. Methods for envelope and instantaneous frequency estimation: (a) Down-conversion method; (b) RF signal based method.

1) Envelope Detection: From physical point of view, the determination of the envelope of an impulse radio signal is a well-known operation [28], [29], but an energy detection is often targeted [30]. Here the aim is to obtain an accurate measurement of the envelope. To model an envelope tracking system behavior, it is chosen to define the envelope as the linear interpolation between each extrema of the oscillating pulse signal from mathematical point of view. However, this technique restricts the kind of impulse signals which can be measured. Indeed there is a trade-off between the bandwidth and the oscillation frequency inside the pulse. In order to have a good approach of the envelope with linear interpolation between each extrema of the oscillating pulse, it is necessary to have enough oscillations inside the pulse with a sufficiently long duration. As said in Section 6.8a.12.1, "Transmitter specification," of the IEEE 802.15.4a Standard [7], the pulse shape is not prescribed but has to comply with the pulse duration defined for the root raised cosine reference pulse across their cross-correlation. For example, for a 2 ns duration for the reference pulse, the 8th order Butterworth pulse used is existing during $5 \mathrm{~ns}$, whereas achieving envelope extraction of pulse presented in [31] and [32] seems more difficult with this technique.

2) Instantaneous Frequency Estimation: The measurement of the instantaneous frequency of an oscillating signal attracts great interests for a long time. A digital instantaneous frequency measurement subsystem was presented in 1996 in [33]. It is based on a mix between the RF signal and the delayed one. This idea is taken again in 2007 in [34] to build a phase-noise measurement circuit. Based on FIR filters, the instantaneous frequency estimator presented in [35] for FMtelemetry applications explains how to digitally extract the instantaneous frequency from a discrete-time baseband complex signal. Below the continuous time methods based on the delayed signal, as in [33], is applied to our impulse signal to get the instantaneous frequency.

From the real oscillating signal of (3), a new signal $s_{\tau}$ (Fig. 5) is built by time delay operation:

$$
s_{\tau}(t)=s(t-\tau)=a(t-\tau) \cos \left(2 \pi v_{0}(t-\tau)+\varphi(t-\tau)\right) .
$$


The product of $s$ and $s_{\tau}$ gives the mix signal:

$$
\begin{aligned}
s_{\text {mix }}(t)= & \frac{a(t) a(t-\tau)}{2}\left[\cos \left(2 \pi \nu_{0} \tau+\varphi(t)-\varphi(t-\tau)\right)\right. \\
& \left.+\cos \left(2 \pi v_{0}(2 t-\tau)+\varphi(t)+\varphi(t-\tau)\right)\right] .
\end{aligned}
$$

Then the delay $\tau$ is chosen in order to have:

$$
2 \pi v_{0} \tau=\frac{\pi}{2}+2 k \pi, k \in \mathbb{N} \text {. }
$$

With the condition (11), due to trigonometric transformation $s_{\text {mix }}(t)$ becomes:

$$
\begin{aligned}
s_{\text {mix }}(t)= & -\frac{a(t) a(t-\tau)}{2}[\sin (\varphi(t)-\varphi(t-\tau)) \\
& \left.+\sin \left(2 \pi v_{0} 2 t+\varphi(t)+\varphi(t-\tau)\right)\right] .
\end{aligned}
$$

Here, if the difference $\varphi(t)-\varphi(t-\tau)$ is very close to zero (it means slow variations for the instantaneous phase),

$$
\sin (\varphi(t)-\varphi(t-\tau)) \approx \varphi(t)-\varphi(t-\tau) .
$$

Combining (12) and (13) and using a low pass filtering to remove high frequency components, the instantaneous phase can be computed. When the signal $s_{\text {out }}(t)$ (Fig. 5) is divided by the product of the envelope and the delayed envelope, the result is:

$$
\frac{s_{\text {out }}(t)}{a(t) a(t-\tau)} \approx-\frac{1}{2}(\varphi(t)-\varphi(t-\tau)) .
$$

Here, the definition (4) of the instantaneous frequency is not so far. As shown in Fig. 5, the result in (14) is divided by the delay $\tau$ and the estimation gives:

$$
f(t) \approx-\frac{1}{a(t) a(t-\tau)} \frac{s_{\text {out }}(t)}{\tau \pi} .
$$

\section{Comparison of the Two Methods}

The accuracy of both studied methods is verified here. The down-conversion method for the estimation of the time domain waveforms presented in Fig. 5 is only based on wellknown mathematical operations to estimate $a(t)$ and $f(t)$. This method is chosen as a reference in order to evaluate the RF signal based method. Note that for the rest of the study the pulse was measured with a time step $\Delta t$ of 25 ps since the maximum available sampling frequency was $40 \mathrm{GHz}$. A number of 643 points was measured in time domain so the time vector has a duration of $16.05 \mathrm{~ns}$, but inside the pulse only 164 points were measured above the threshold defined in Fig. 3. The estimated central frequency is $3943 \mathrm{MHz}$ and the corresponding $\tau$ is $63 \mathrm{ps}$. The frequency step corresponding to the simulation duration is $62.3 \mathrm{MHz}$.

In Fig. 6 the envelope of the impulse signal is drawn thanks to the methods introduced in Section III. Both of the envelopes are quite identical.

In Fig. 7, the instantaneous frequency from the downconversion method and from the RF signal based method are shown. Both signals vary in the same way with almost identical oscillation and amplitude values. Thus, both methods are matching. Note that hatched windows are drawn to highlight time periods where the impulse signal contains only noise so

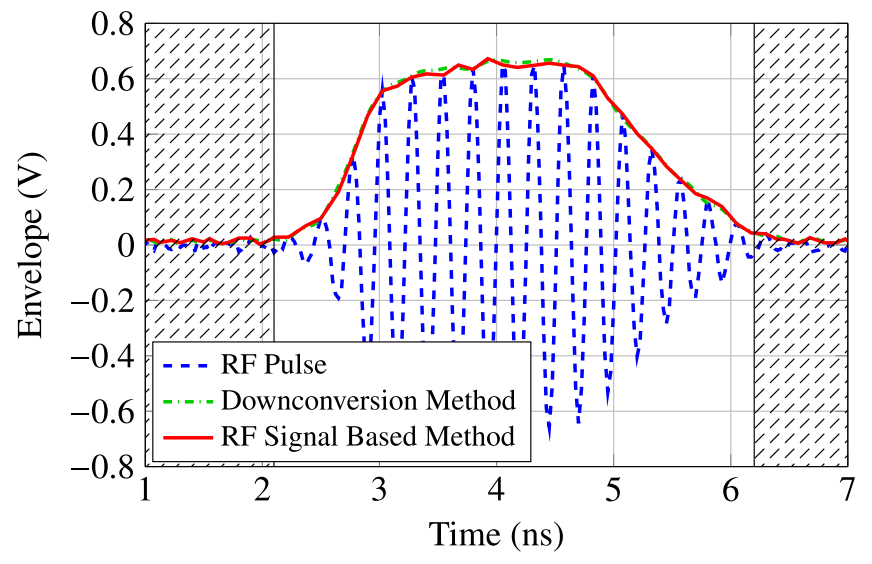

Fig. 6. Envelope for a pulse from an IR-UWB transmitter [26].

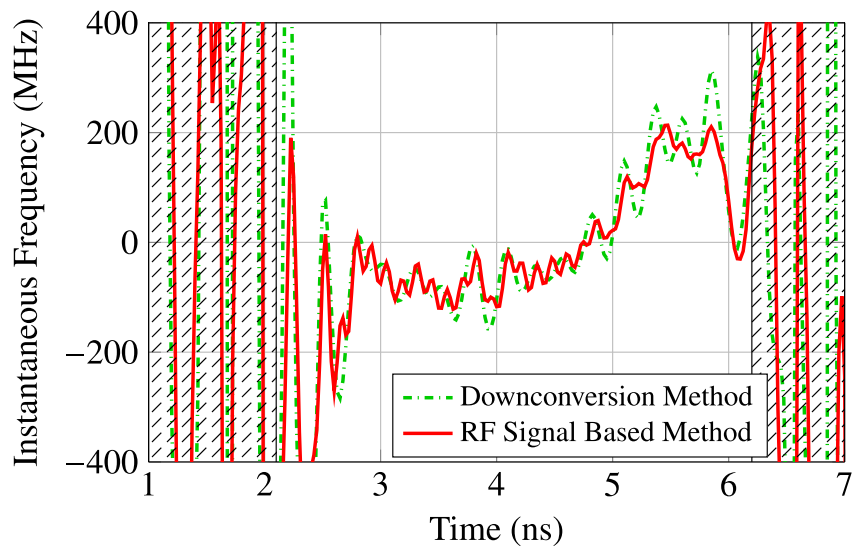

Fig. 7. Instantaneous frequency for a pulse from an IR-UWB transmitter [26].

the definition of the instantaneous frequency does not make sense.

For the rest of this study, the RF signal based method was chosen in order to estimate the time parameters because this method offers more practical possibilities for a future hardware implementation of an on-chip measurement system. Even if the down-conversion method seems interesting, the need of local oscillator to provide the I and Q baseband signal gives lots of constraints for hardware implementation, especially in terms of silicon area and power consumption. Based on a direct measurement of RF signal, the second method does not have these drawbacks. Nevertheless, the RF signal based method still should use a technique that allows to estimate the carrier frequency $v_{0}$ of the pulse in order to have a reliable result for the estimation of the delay $\tau$. It is important to work in the frequency baseband in order to reduce the constraints on the analog-to-digital conversion, which can be very expensive in terms of power and area costs. Moreover with a maximum of analog hardware implementation for time parameters extraction it is possible to reduce the complexity of the digital baseband implementation in order to avoid adding additional digital part around transmitters. 


\section{ESTABLishment OF A FREQUENCY DOMAIN REPRESENTATION}

In order to approach the result of the Fourier transform, the aim of this section is to get a frequency representation which would not require an analytical knowledge of the signal envelope and/or its instantaneous phase. Thanks to a frequency representation based on the time domain parameters, it would be possible to find which time part of the time domain signal has the major contribution in the PSD in the frequency domain.

\section{A. Time Domain Baseband Signal Expression}

Even if it is possible to compute the power spectral density with the Fourier transform, it is not suitable to use the DFT because the problem is that the time-to-frequency link is lost and it is not possible to understand in which manner both time domain waveforms act on the result. To implement the control in the time domain seems to be more reliable on hardware level. Therefore, the attention is focused on approximation of the envelope and the instantaneous frequency of the baseband signal.

Indeed, from practical point of view, $a(t)$ and $f(t)$ (at least $\varphi(t))$ are physically measurable characteristics. Here the only hypothesis is that $a(t)$ and $\varphi(t)$ can be approximated by piece-wise linear or non-linear functions. For an nth-order approximation, the time error $\epsilon_{n}$ between the signal $s(t)$ and the approximate signal $s_{n}(t)$ and its norm as a squareintegrable function $\left(\mathcal{L}^{2}\right.$-norm $)$ are:

$$
\begin{aligned}
\epsilon_{n}(t) & =s(t)-s_{n}(t) \\
\left\|\epsilon_{n}\right\|_{2} & =\sqrt{\int_{\mathbb{R}}\left|\epsilon_{n}(t)\right|^{2} d t}
\end{aligned}
$$

It is well-known that the $\mathcal{L}^{2}$-norm of the error decreases when the approximation order $n$ is increased. Moreover, according to the Parseval's identity, the $\mathcal{L}^{2}$-norm of $\epsilon_{n}$ is the same in time than in frequency domain. That is why it would be better to use the time domain signal model which would make the best approximation in order to get an accurate PSD.

Thus, to realize these approximations, a piece-wise approximation can be used for $a(t)$ and $f(t)$ using polynomial functions fitting to the waveforms. However, the continuous polynomial approach with 2 nd order or more requires difficult computations to find the analytical expression of Fourier transform. In order to reduce the problem complexity, it is possible to use a continuous linear time variant piece-wise model for both parameters $a(t)$ and $f(t)$. However, it leads to second order Fresnel integration into the Fourier transform calculation because $\varphi(t)$ is the primitive of $f(t)$. A compromise can be found if one can tolerate the consideration of the linear evolution for the phase, thus a piecewise constant behavior of the instantaneous frequency. The envelope will keep a linear model in a first approach. Thus a finite sum of rectangular window $\Pi$ linearly approximates the signal $s_{B B}(t)$ by

$$
s_{B B_{\text {linear }}}(t)=\sum_{i=0}^{N-1} a_{i}(t) \mathrm{e}^{j \varphi_{i}(t)} \Pi_{\Delta t}\left(t-\frac{\Delta t(2 i+1)}{2}\right),
$$

where

$$
\begin{aligned}
& a_{i}(t)=b_{i} t+c_{i}, \\
& \varphi_{i}(t)=2 \pi f_{i} t+\varphi_{0, i},
\end{aligned}
$$

where $\Delta t$ is the chosen length of one time window and $N$ is the number of intervals according to the chosen unitary time step $\Delta t\left(N=T_{p} / \Delta t\right)$. The minimum value for $\Delta t$ is the minimum time step enabled by the measurement device. The terms $b_{i}$ and $f_{i}$ are respectively the $\mathrm{i}$ th slope value of envelope $a(t)$ and instantaneous frequency $f(t)$ in the piece-wise approximation; $c_{i}$ and $\varphi_{0, i}$ are the y-intercept of each linear part. Moreover $\varphi_{0, i}$ insures the continuity during the $f(t)$ integration to obtain the phase $\varphi(t)$ of the signal according to (4).

It can be also interesting to consider the envelope with a piece-wise time invariant approach. It gives:

$$
s_{B B_{\text {stairs }}}(t)=\sum_{i=0}^{N-1} a_{i} \mathrm{e}^{j \varphi_{i}(t)} \Pi_{\Delta t}\left(t-\frac{\Delta t(2 i+1)}{2}\right) .
$$

Here $a_{i}$ is the $\mathrm{i}^{\text {th }}$ sample of envelope $a(t)$.

\section{B. Spectral Study}

The definition in (18) assumes that $s_{B B}(t)$ is a finite energy signal like impulse signals. As a square-integrable function, it is now possible to determine its Fourier transform:

$$
\begin{aligned}
S_{B B}^{l i n}(v)=\sum_{i=1}^{N} \mathrm{e}^{j \varphi_{0, i}} & {\left[b _ { i } \left(\frac{t_{i} \mathrm{e}^{-j 2 \pi\left(\nu-f_{i}\right) t_{i}}-t_{i-1} \mathrm{e}^{-j 2 \pi\left(\nu-f_{i}\right) t_{i-1}}}{-j 2 \pi\left(v-f_{i}\right)}\right.\right.} \\
& \left.-\frac{\mathrm{e}^{-j 2 \pi\left(\nu-f_{i}\right) t_{i}}-\mathrm{e}^{-j 2 \pi\left(\nu-f_{i}\right) t_{i-1}}}{-4 \pi^{2}\left(v-f_{i}\right)^{2}}\right) \\
& \left.+c_{i} \Delta t \operatorname{sinc}\left(\pi\left(v-f_{i}\right) \Delta t\right) \mathrm{e}^{j \theta_{i}(v)}\right],
\end{aligned}
$$

where

$$
\theta_{i}(v)=-2 \pi\left(v-f_{i}\right) \frac{\Delta t(2 i+1)}{2}+\varphi_{0, i}
$$

The delayed rectangular windows yield cardinal sine functions in frequency domain which are multiplied by a complex exponential function depending of the delay applied on the different windows. In (20), the envelope which is approximated by linear piece-wise functions, produces in the frequency domain two complex quotients between complex exponential functions and the frequency variable $v$. It is interesting to note that those quotients are similar to a cardinal sine. Finally, the instantaneous phase of the impulse signal, represented by the time dependent complex exponential function, provides a Dirac delta function which modulates the cardinal sine functions in frequency domain.

In the same way, it is possible to calculate the Fourier transform of the signal from the model in (19) with piecewise time constant approach for the envelope:

$$
S_{B B}(v)=\sum_{i=0}^{N-1} a_{i} \Delta t \operatorname{sinc}\left(\pi\left(v-f_{i}\right) \Delta t\right) \mathrm{e}^{j \theta_{i}(v)} .
$$


As mentioned before, in order to estimate the PSD of the train of pulses $x(t)$ thanks to the Bennett's formula for instance, the squared modulus of the Fourier transform of the emitted pulse $s(t)$ is necessary. Indeed, for instance, the impulse modulation for the signal can be:

$$
x(t)=\sum_{i} \alpha_{i} s\left(t-i T_{s}\right),
$$

where $s(t)$ is the emitted pulse, $T_{s}$ is the symbol duration and $\alpha_{i}$ is the symbol defining the weighting of the amplitude at the instant $i T_{s}$. For instance, for a linear modulation with independent symbols, the PSD of the train of pulses is:

$$
\begin{aligned}
P S D_{x}= & \frac{\sigma_{a}^{2}}{T_{S}}\left|S_{B B}(v)\right|^{2} \\
& +\frac{\mu_{a}^{2}}{T_{s}^{2}} \sum_{i}\left|S_{B B}\left(\frac{i}{T_{s}}\right)\right|^{2} \delta\left(v-\frac{i}{T_{s}}\right),
\end{aligned}
$$

where $\sigma_{a}$ and $\mu_{a}$ are respectively the mean value and the variance of the whole of the symbols $\alpha_{i}$. For convenience, just the result from the Fourier transform in (22) is given:

$$
\begin{aligned}
\left|S_{B B}(v)\right|^{2}= & {\left[\left(\sum_{i=0}^{N-1} r_{i}(v) \cos \left(\theta_{i}(v)\right)\right)^{2}\right.} \\
& \left.+\left(\sum_{i=0}^{N-1} r_{i}(v) \sin \left(\theta_{i}(v)\right)\right)^{2}\right],
\end{aligned}
$$

where $\theta_{i}(v)$ is defined in (21) and

$$
r_{i}(v)=a_{i} \Delta t \sin _{c}\left(\pi\left(v-f_{i}\right) \Delta t\right) .
$$

Each part in the piece-wise approximation of $a(t)$ and $f(t)$ displays a radius function $r_{i}$ and an angle function $\theta_{i}$. The first has a maximum value which is equal to $a_{i} \Delta t$ at the instantaneous frequency value $f_{i}$ due to the presence of the cardinal sine function. The width of each cardinal sine function is inversely proportional to $\Delta t$ interval in the piecewise approximation. In practical cases, this cardinal sine function has no impact on the signal at low frequency. However when the number of samples is low due to a large $\Delta t$ interval, the sine cardinal functions enable to improve the precision of the signal.

\section{Frequency Domain Estimation Method Validation}

The discrete Fourier transform of the oscillating pulse can be obtained from the MATLAB fast Fourier transform (FFT) algorithm. In order to evaluate the accuracy of equation (25), this FFT result is used as the reference. In order to get higher frequency resolution, the time periods when the pulse is switched off are retained during the computation. Also it avoids any problem due to the time repetition of the signal given by FFT algorithm.

An example of frequency domain representation obtained from piece-wise time constant approach of time domain waveforms shown in Figs. 6 and 7 is given in Fig. 8. The aim is to prove the validity of the proposed frequency representation

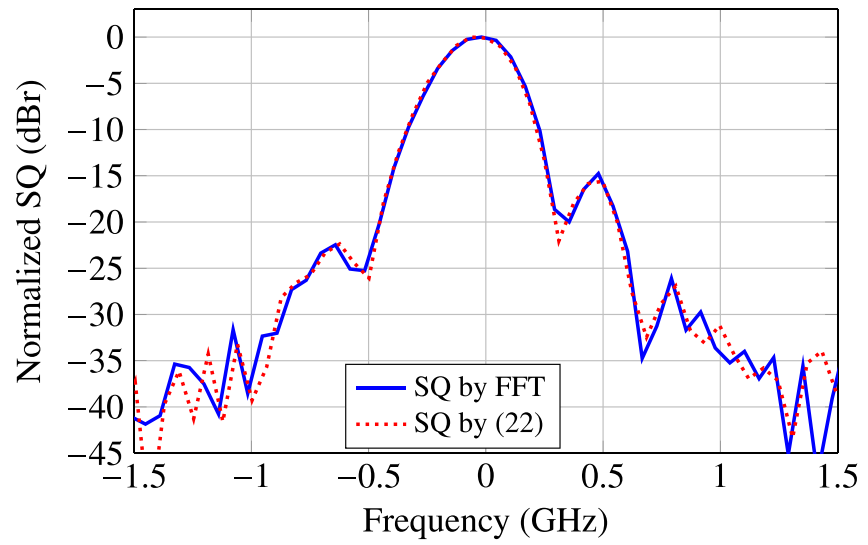

Fig. 8. Comparison between DFT and the proposed method results.

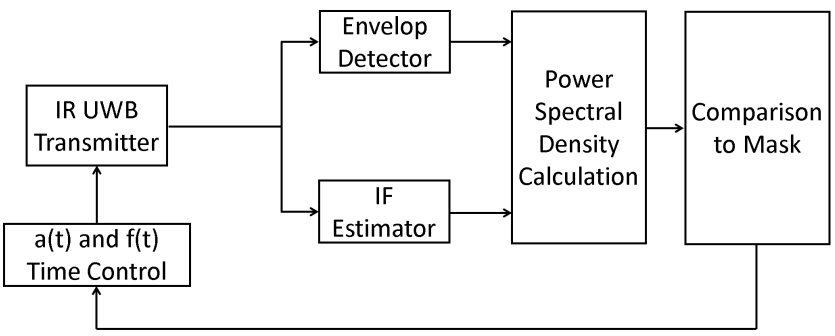

Fig. 9. Method to estimate the PSD of an IR UWB transmitter.

so the continuous part of (24), called for the rest of the study SQ (for Square Modulus), is just represented and:

$$
S Q(v)=\frac{1}{T_{S}}\left|S_{B B}(v)\right|^{2} .
$$

The SQ can be seen as the power spectral density of the emitted pulse. In Fig. 8 the dotted graph $S_{B B}(v)$ is the result of (27) while the solid line is calculated thanks to FFT algorithm. The sampling frequency is still $40 \mathrm{GHz}$ but the results are only shown on the frequency baseband ([-1.5 GHz; $1.5 \mathrm{GHz}]$ ) and are normalized since the power limits in IEEE 802.15.4a Standard are given in $\mathrm{dB}$ relative to the maximum spectral density of the signal.

Both methods yield the same result on the "low" frequency band (about $[-1 \mathrm{GHz} ; 1 \mathrm{GHz}]$ ) of the baseband spectrum. Some differences appear above this band, especially on the deep value in the spectrum, but those values are not essential for the comparison to the PSD masks.

\section{Perspective For PSD Correction}

The aim of this section is to validate the previous analytical expressions by applying them to a measured pulse generated with an existing IR UWB transmitter [26].

Section IV-B has shown how time parameters $a(t)$ and $f(t)$ influence the emission power in frequency domain. The method described in Fig. 9 is now applied to make a PSD compliant with the IEEE 802.15.4a mask shown in Fig. 1 by accurate modifications of the pulse thanks to the knowledge of time-to-frequency link. Indeed, after the extraction of time parameters $a(t)$ and $f(t)$ with the RF signal based method, 

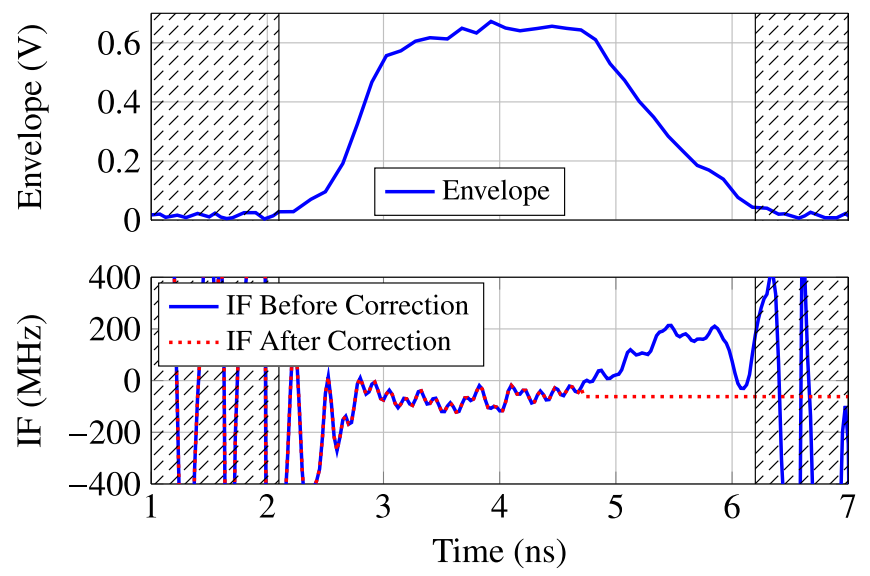

Fig. 10. Envelope and Instantaneous Frequency before and after IF correction.

the analytical expression in (25) is used to introduce a simple strategy in the time domain in order to modify the SQ curve according to the targeted mask. For convenience, the modulation in the pulse repetition is not taken into account. An average of the emitted pulses during one millisecond period using one megahertz resolution bandwith is required by the IEEE 802.15.4a Standard. Here, the possibility to control precisely the envelope and the instantaneous frequency is considered because the study was done on a measured pulse but in an integrated transmitter system the inputs would act on the oscillating output so the response of the transmitter to its inputs should be well-known.

The SQ drawn in Fig. 8 presents a secondary lobe in positive frequencies around $500 \mathrm{MHz}$ which is higher than the one in negative frequencies. This lobe does not comply with frequency mask. Two solutions to reduce the power density of this lobe are suggested.

The first solution is to modify the instantaneous frequency. In Fig. 10, the SQ before correction shows positive values which appeared during the pulse extinction and included between 0 and $300 \mathrm{MHz}$. From the expression of $r_{i}(v)$, those values of instantaneous frequencies engender some energy on the right side of the SQ. For instance a part of this energy can be moved to the main lobe. To do that, the instantaneous frequency values from $4.7 \mathrm{~ns}$ up to the pulse end are arbitrarily replaced to the mean value of the instantaneous frequency between 3 and $4.7 \mathrm{~ns}$, so $-70 \mathrm{MHz}$. The SQ obtained is shown in Fig. 11 and indicates a reduction of the positive secondary lobe after the correction of the instantaneous frequency. So by correction on the instantaneous frequency it is possible to move some energy of the pulse along the frequency axis. Note, by adding values close to the carrier frequency into the instantaneous frequency, the maximum of the main lobe of the SQ is shifted to negative frequencies. Thus the modification produced on the whole SQ by changing a part of the instantaneous frequency have to be taken into account in a calibration strategy. On the SQ without normalization a reduction of $1 \mathrm{~dB}$ was noticed.

A second solution to reduce the power density of a lobe can be considered by modifying the envelope. Indeed, as seen before, the instantaneous frequency gives the distribution of

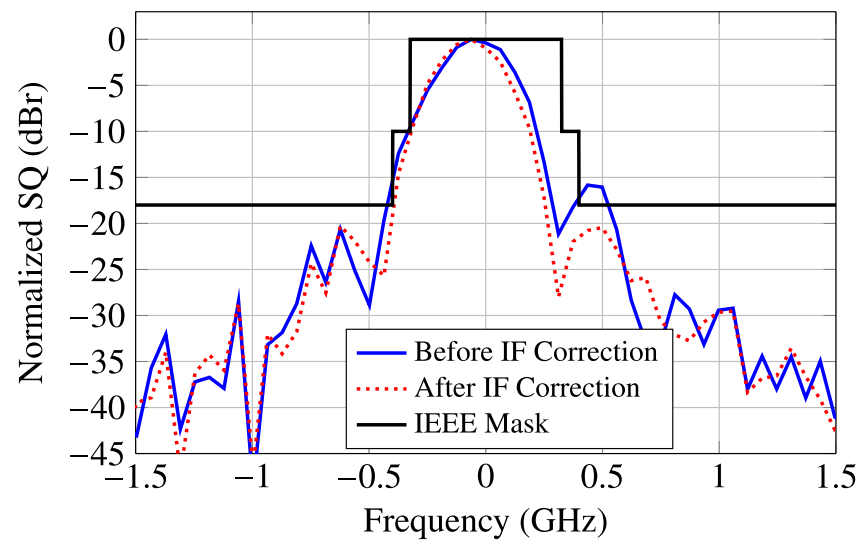

Fig. 11. Comparison before and after IF correction.
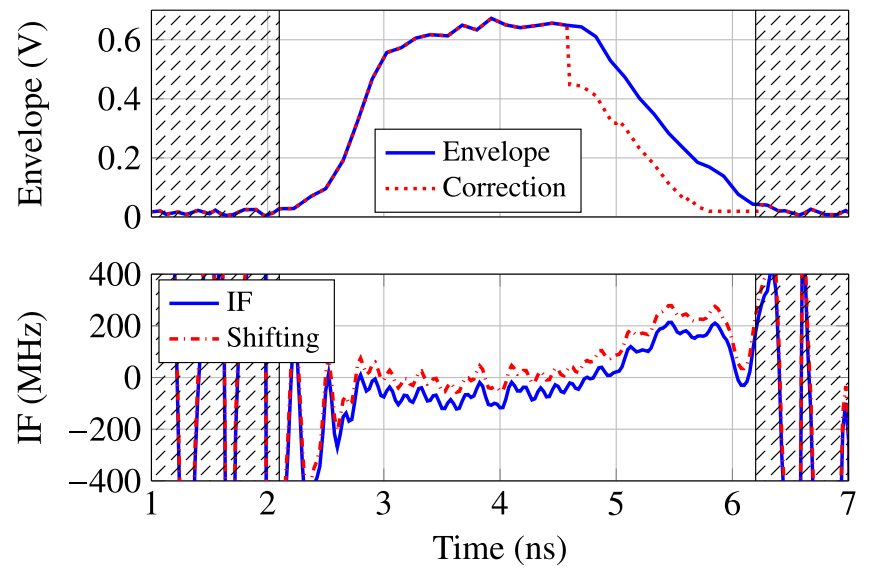

Fig. 12. Envelope and instantaneous frequency before and after envelope correction.

the energy along the frequency axis. But according to (22), the values of the envelopes gives the amount of energy. Thus, in order to reduce the contribution of these instantaneous frequency values, it is also possible to act on the envelope values. However, to reduce the envelope where the instantaneous frequency is positive can also increase the PSD in negative frequencies because the PSD at the specific frequency $v$ is the squared modulus of a complex vectorial sum. So the influence of the $\mathrm{i}$-th vector in the sum is not the same according the frequency $v$.

The result obtained with this second solution is represented in Fig. 12. The envelope between 4.5 and 6 ns has been reduced by subtracting a constant value. The result after the envelope correction is shown in Fig. 13. The secondary lobe in positive frequencies is actually lowered and respects the mask. But in this case, the reduction of the envelope during the extinction of the pulse has an effect on the bandwidth of the main lobe of the SQ too and the maximum is not only shifted on the frequency axis. To shift back all the SQ into the mask, it is possible to make a shift on the carrier frequency by adding the same positive correcting value on all the instantaneous frequencies.

The link between these two time characteristics and the origin of the spectral asymmetry is established and these 


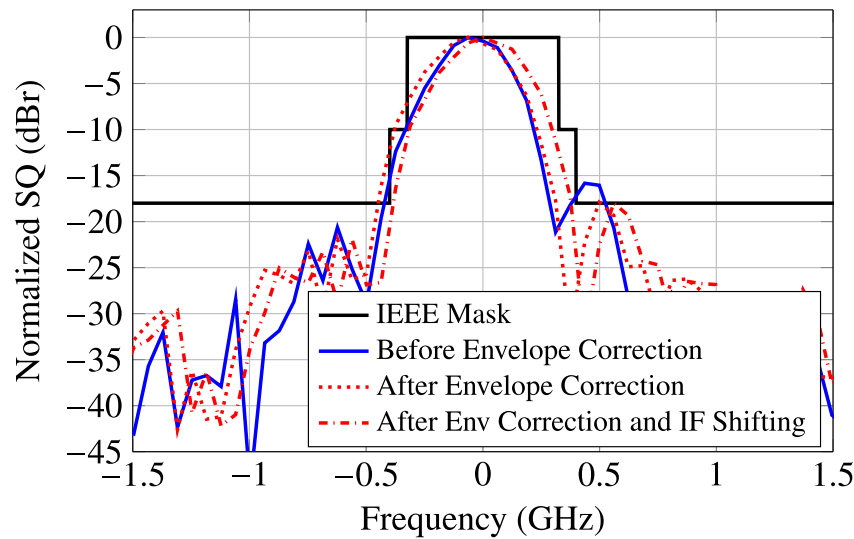

Fig. 13. Comparison before and after envelope correction.

examples demonstrate the possibility to modify the SQ by using the envelope and the instantaneous frequency thanks to (25). But, in order to highlight the possibility to act on the PSD thanks to the envelope and instantaneous frequency, to perform all the previous corrections made on these two time parameters, it was assumed that it is possible to act on these time domain waveforms independently. However, across (15), if the envelope is modified, the instantaneous frequency has to be extracted again. Moreover, according to (25), for a given value of frequency $v$, every value of the envelope and the instantaneous frequency take part in the value of the SQ on this frequency point $v$. Also there is no orthogonal relation in order to tune the frequency domain from the time domain. It is possible to use an input sweep method that consists in trying a predefined configurations of input knobs. The calibration should act on the inputs of the transmitter step-bystep, learning each time the effect of its previous time domain correction on the PSD. This machine learning method could be very time and energy consuming, but since it is used once or very few times in the calibration mode, it will represent very low amount of the circuit total consumption on the whole functional period.

\section{CONCLUSION}

One of the main challenges for IR-UWB transmitters is to comply with IEEE 802.15.4a Standard spectral masks while these emitters are disturbed by process, voltage, and temperature variations. In this way self-calibration systems have to be integrated to ultra-wideband impulse radio transmitters which implies on-chip power spectral density estimation. It requires the computation of the Fourier transform of the emitted pulse but it is a difficult task due to the impulse nature of the signal. The method presented here is based on the direct extraction from the RF signal of the envelope and the instantaneous frequency. With these two time domain waveforms, it is possible to fully represent the power spectral density around the zero frequency. A piece-wise approach of these time domain waveforms is proposed in order to allow the estimation of a frequency domain representation of the impulse signal. This approach is independent of the pulse shape in order to be used with different IR-UWB transmitters. The analytical expression of the Fourier transform of the emitted pulse shows how the time domain waveforms influence the frequency domain. In this way it is possible to modify the envelope and the instantaneous frequency in order to comply with IEEE 802.15.4a Standard spectral masks. This result and the well-known continuous wave techniques employed to extract the time domain parameters prove the possibility of the hardware implementation of on-chip calibration systems for IR-UWB transmitters.

\section{REFERENCES}

[1] O. R. Sparrow et al., "High rate UWB CMOS transceiver chipset for WBAN and biomedical applications," Analog Integr. Circuits Signal Process., vol. 81, no. 1, pp. 215-227, Oct. 2014.

[2] R. Vauche et al., "High efficiency UWB pulse generator for ultra-lowpower applications," Int. J. Microw. Wireless Technol., vol. 8, no. 3, pp. 495-503, May 2016.

[3] S. Roy, J. R. Foerster, V. S. Somayazulu, and D. G. Leeper, "Ultrawideband radio design: The promise of high-speed, short-range wireless connectivity," Proc. IEEE, vol. 92, no. 2, pp. 295-311, Feb. 2004.

[4] S. Geng, D. Liu, Y. Li, H. Zhuo, W. Rhee, and Z. Wang, "9.2 A 13.3mW $500 \mathrm{Mb} / \mathrm{s}$ IR-UWB transceiver with link-margin enhancement technique for meter-range communications," in IEEE Int. Solid-State Circuits Conf. Dig. Tech. Papers (ISSCC), Feb. 2014, pp. 160-161.

[5] D. Dardari, A. Conti, U. Ferner, A. Giorgetti, and M. Z. Win, "Ranging with ultrawide bandwidth signals in multipath environments," Proc. IEEE, vol. 97, no. 2, pp. 404-426, Feb. 2009.

[6] Revision of Part 15 of the Commission's Rules Regarding UltraWideband Transmission Systems, document ET-Docket FCC, 2002.

[7] IEEE Standard for Information Technology-Telecommunications and Information Exchange Between Systems-Local and Metropolitan Area Networks-Specific Requirement Part 15.4: Wireless Medium Access Control (MAC) and Physical Layer (PHY) Specifications for Low-Rate Wireless Personal Area Networks (WPANs), IEEE Standard 802.15.4a2007 (Amendment to IEEE Standard 802.15.4-2006), 2007, pp. 1-203.

[8] J. B. Radic, A. Djugova, L. F. Nagy, and M. S. V. Misic, "A 3.1-6.65 GHz, $933 \mu \mathrm{W}$ impulse radio pulse generator with tuneable spectrum in $0.18 \mu \mathrm{m}$ CMOS," in Proc. Telecommun. Modern Satellite, Cable Broadcast. Services (TELSIKS), vol. 2. Oct. 2013, pp. 378-382.

[9] "Power spectrum estimation," Nat. Semicond., Santa Clara, CA, USA, Tech. Rep. 255, Nov. 1980.

[10] M. Z. Win, "Spectral density of random time-hopping spread-spectrum UWB signals with uniform timing jitter," in Proc. IEEE Military Commun. Conf. (MILCOM), vol. 2. Nov. 1999, pp. 1196-1200.

[11] S. Majhi, A. S. Madhukumar, Y. Nasser, and J. F. Helard, "Power spectral analysis of orthogonal pulse-based TH-UWB signals," in Proc. IEEE 71st Veh. Technol. Conf. (VTC-Spring), May 2010, pp. 1-5.

[12] J. G. Proakis, "Characterization of communication signals and systems," in Digital Communications (Electrical and Computer Engineering), 4th ed. Boston, MA, USA: McGraw-Hill, 2007, ch. 4, pp. 148-222.

[13] H. Sheng, P. Orlik, A. M. Haimovich, L. J. Cimini, and J. Zhang, "On the spectral and power requirements for ultra-wideband transmission," in Proc. IEEE Int. Conf. Commun. (ICC), vol. 1. May 2003, pp. 738-742.

[14] W. Gao, R. Venkatesan, and C. Li, "A pulse shape design method for ultra-wideband communications," in Proc. IEEE Wireless Commun. Netw. Conf. (WCNC), Mar. 2007, pp. 2800-2805.

[15] J. Hu, T. Jiang, Z. Cui, and Y. Hou, "Design of UWB pulses based on Gaussian pulse," in Proc. 3rd IEEE Int. Conf. Nano/Micro Eng. Molecular Syst., Jan. 2008, pp. 651-655.

[16] B. Hu and N. C. Beaulieu, "Pulse shapes for ultrawideband communication systems," IEEE Trans. Wireless Commun., vol. 4, no. 4, pp. 1789-1797, Jul. 2005.

[17] D. Adhikari and C. Bhattacharya, "Power spectral density of modified Hermite pulses for M-ary pulse shape modulation," in Proc. Int. Conf. Commun. Devices Intell. Syst. (CODIS), Dec. 2012, pp. 13-16.

[18] S. Gezici, Z. Sahinoglu, H. Kobayashi, and H. V. Poor, "Ultra-wideband impulse radio systems with multiple pulse types," IEEE J. Sel. Areas Commun., vol. 24, no. 4, pp. 892-898, Apr. 2006.

[19] X. Liu, A. B. Premkumar, and A. S. Madhukumar, "Pulse shaping functions for UWB systems," IEEE Trans. Wireless Commun., vol. 7 , no. 5, pp. 1512-1516, May 2008

[20] V. Goyal and B. S. Dhaliwal, "Optimal pulse generation for the improvement of ultra wideband system performance," in Proc. IEEE Recent Adv. Eng. Comput. Sci. (RAECS), Mar. 2014, pp. 1-6. 
[21] Z. Bai, J. Liu, and H.-H. Chen, "Design of ultra-wideband pulses based on spectrum shifted Gaussian waveforms," IET Commun., vol. 7, no. 6, pp. 512-520, Apr. 2013.

[22] M. B. Menon, A. Gopakumar, and N. V. Iqbal, "A hybrid approach for UWB pulse shaping," in Proc. 2nd Int. Conf. Electron. Commun. Syst. (ICECS), Feb. 2015, pp. 373-377.

[23] S. H. I. Barboza, J. A. A. Palacio, E. Pontes, and S. T. Kofuji, "Fifth derivative Gaussian pulse generator for UWB breast cancer detection system," in Proc. IEEE Int. Conf. Ultra-WideBand (ICUWB), Sep. 2014, pp. 269-273.

[24] R. Vauche, S. Bourdel, N. Dehaese, O. Fourquin, and J. Gaubert, "Fully tunable UWB pulse generator with zero DC power consumption," in Proc. IEEE Int. Conf. Ultra-Wideband (ICUWB) Sep. 2009, pp. $418-422$.

[25] I. Barraj, H. Trabelsi, and M. Masmoudi, "A new UWB pulse shaping for IEEE 802.15.4a," in Proc. 10th Int. Multi-Conf. Syst. Signals Devices (SSD), Mar. 2013, pp. 1-5.

[26] G. Masson, F. Hameau, and L. Ouvry, "UWB pulse generator," WO Patent 2015/091668 A1, Jun. 25, 2015.

[27] J. Li, W. Rhee, and Z. Wang, "A dual-carrier IR-based UWB transmitter with improved spectral efficiency," in Proc. Int. Conf. Commun. Circuits Syst. (ICCCAS), Jul. 2009, pp. 788-792.

[28] M. K. Kang and T. W. Kim, "CMOS IR-UWB receiver for $\pm 9.7-\mathrm{mm}$ range finding in a multipath environment," IEEE Trans. Circuits Syst. II, Exp. Briefs, vol. 59, no. 9, pp. 538-542, Sep. 2012.

[29] M. A. K. Jazairli and D. Flandre, "An ultra-low-power UWB IR pulse receiver using 65nm CMOS technology," in Proc. IEEE Faible Tension Faible Consommation (FTFC), Jun. 2013, pp. 1-4.

[30] N. Dehaese et al., "Low-power CMOS energy detector for noncoherent impulse-radio UWB receivers," in Proc. IEEE Int. Conf. UltraWideband (ICUWB), Sep. 2010, pp. 1-4.

[31] M. Demirkan and R. R. Spencer, "A pulse-based ultra-wideband transmitter in 90-nm CMOS for WPANs," IEEE J. Solid-State Circuits, vol. 43, no. 12, pp. 2820-2828, Dec. 2008.

[32] J. Li, B. Zhou, Y. Sun, W. Rhee, and Z. Wang, "Reconfigurable, spectrally efficient, high data rate IR-UWB transmitter design using a $\delta$ - $\sigma$ PLL driven ILO and a 7-tap FIR filter," in Proc. Int. Symp. VLSI Design Autom. Test (VLSI-DAT), Apr. 2011, pp. 1-4.

[33] G.-C. Liang, C.-F. Shih, R. S. Withers, B. F. Cole, and M. E. Johansson, "Space-qualified superconductive digital instantaneous frequency-measurement subsystem," IEEE Trans. Microw. Theory Techn., vol. 44, no. 7, pp. 1289-1299, Jul. 1996

[34] W. Khalil, B. Bakkaloglu, and S. Kiaei, "A self-calibrated on-chip phase-noise measurement circuit with $-75 \mathrm{dBc}$ single-tone sensitivity at $100 \mathrm{kHz}$ offset," IEEE J. Solid-State Circuits, vol. 42, no. 12, pp. 2758-2765, Dec. 2007.

[35] E. Hermanowicz, M. Rojewski, G. D. Cain, and A. Tarczynski, "On an instantaneous frequency estimator with FIR filters having maximally flat frequency response error magnitude," Signal Process., vol. 81, no. 7 , pp. 1491-1501, Jul. 2001

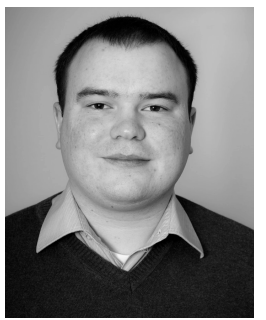

Anthony Goavec received the M.Eng. degree from the Institut National Polytechnique, Grenoble, France. $\mathrm{He}$ is currently working toward the Ph.D. degree with the CEA-Commissariat à l'Energie Atomique et aux Energies Alternatives, Grenoble, France, and with the University of Provence, Aix-Marseille I, France. His current field of research is in the spectral auto-calibration of IR-UWB transmitter. His research interests include RF, spectrum analysis, power amplifier integrated circuits, and analog design in silicon technologies.

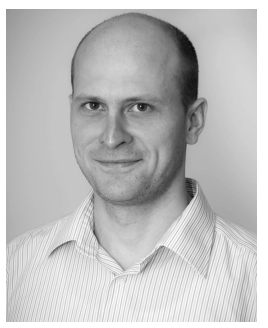

Mykhailo Zarudniev received the M.S. degree in 2008 from the National Technical University of Ukraine "Kiev Polytechnic Institute," Kyiv, Ukraine, and the Ph.D. degree in automatic control from Lyon University, France, in January 2013. He is currently a Research Engineer with the Commissariat à l'Energie Atomique et aux Energies Alternatives, France. His research interests include robust control, identification for control and signal processing with emphasis on the integrated time efficient circuit design methodology.

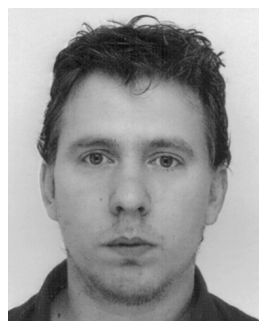

Rémy Vauché got into preparation for the competitive entrance examinations to French Engineering Schools, Metz, France, in 2003. He received the M.Eng. degree in microelectronics and telecommunication from Polytech Marseille, France, and the M.S. degree in microelectronics and nanoelectronics from Aix-Marseille University, Marseille, France, in 2008. He received the Ph.D. degree in Microelectronics from the University of Provence, AixMarseille I, France, in 2011. From 2011 to 2014, he was a lecturer and researcher in the ISEN French Engineering School, Toulon, France. Since 2014, he is now an Associate Professor with Aix-Marseille University and a member of the Integrated Circuits Design Team from the Provence Nanosciences Microelectronics and Materials Laboratory (IM2NP), Marseille. His current field of research is in the design of UWB emitters IC.

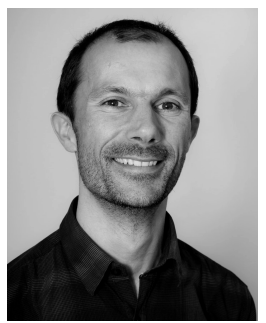

Frédéric Hameau received the M.S. degree in microelectronics from Ecole Nationale Supérieure d'Electronique, d'Informatique et de Radiocommunications de Bordeaux, France, in 2001. He did a 6 month period as RF designer in the STmicroelectronics R\&D Center, Crolles, France, where he designed a power amplifier for Bluetooth applications in $0.12 \mathrm{~m}$ SOI technology. He joined CEALETI Laboratory, France, as RF designer in 2001, where he designed RF frontend blocks using SOI and CMOS technology. He is now in charge of system on chip integration in the wireless sensor network field. His work includes ultra-wide band applications as well as ultra-low power applications.

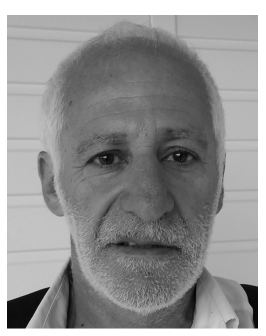

Jean Gaubert received the M.S. and the Ph.D. degrees in applied physics from Paul Sabatier University, Toulouse, France, in 1985 and 1988, respectively. From 1989 to 2001 he was an Assistant Professor at the Ecole Nationale Supérieure de l'Electronique et de ses Applications, Cergy-Pontoise, France, where he was involved with high-speed GaAs and InP bipolar devices modelling. In 2001, he joined Aix-Marseille University (AMU), France, and the Institut Matériaux Microélectronique Nanosciences de Provence (IM2NP UMR CNRS 7334), France, where he has been a full Professor since 2009. His research focuses on the design and integration of RF/Microwave CMOS integrated-circuits and systems for wireless communications.

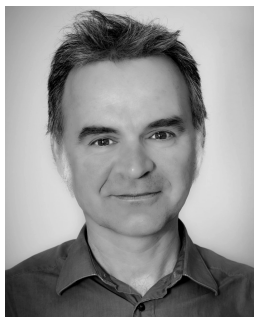

Eric Mercier graduated from the ENSEEIH of Toulouse, France, in 1991, and holds a DEA in microwaves focused on near-field/far-field antenna diagram conversion done at Alcatel Space, Toulouse, France. After having held positions in the optical test equipment with Schlumberger, for physical fiber optical link tests, as analog and signal processing engineer, he has pursued his work in semiconductor companies like ST and Atmel as R\&D Application \& Characterization engineer, as well as Marketing engineer. His main field of interest has been low power RF dedicated to Wireless Sensor Network. He is now at CEA-Leti, France, since 2006, where he has led projects in the scope of ULP RF, with a specific focus on low-power RF transceiver design \& implementation as well as on embedded resources dedicated to low-power WSN solutions. He is now the Head Manager of the Laboratory for Architectures \& Integrated RF design (LAIR). This lab is in charge of designing RF solutions for ULP, UWB, $\mathrm{UNB}, \mathrm{mmW}$, high-data rate, RFID, PA, and FEM, with a common target of addressing the lowest possible power consumption and make use of the most advanced CMOS technologies. He has coauthored some conference papers and participated to a book chapter on the topic of WSN. 